\title{
Heuristic procedures for generating stable project baseline schedules
}

\author{
Stijn Van de Vonder, Erik Demeulemeester and Willy Herroelen \\ Department of Applied Economics, K.U.Leuven, Belgium \\ e-mail: < first name $>$.<family name $>@ e c o n . k u l e u v e n . b e$
}

February 16, 2005

\begin{abstract}
Solution robust project scheduling is a growing research field aiming at constructing proactive schedules to cope with multiple disruptions during project execution. When stochastic activity durations are considered, including time buffers between activities is a proven method to improve the stability of a baseline schedule.

This paper introduces multiple algorithms to include time buffers in a given schedule while a predefined project due date remains respected. Multiple efficient heuristic and meta-heuristic procedures are proposed to allocate buffers throughout the schedule. An extensive simulation-based analysis of the performance of all algorithms is given. The impact of the activity duration variance structure on the performance is discussed in detail.
\end{abstract}

\section{Introduction}

The vast majority of the research efforts in project scheduling over the past several years have concentrated on the development of exact and heuristic procedures for the generation of a workable baseline schedule (pre-schedule or predictive schedule) assuming complete information and a static and deterministic environment. During execution, however, a project may be subject to considerable uncertainty, which may lead to numerous schedule disruptions. Activities can take shorter or longer than primarily expected, resource requirements or availability may vary, new activities might have to be inserted, etc..

Recent research (Van de Vonder et al. 2004a) has demonstrated that when projects have to be executed in the face of uncertainty, proactive-reactive project scheduling procedures are capable of combining schedule stability and makespan performance and the use of an objective function aiming at schedule stability 
pays off. The objective of this paper is to develop and validate a number of heuristic procedures for generating stable project baseline schedules.

The problem used as our vehicle of analysis can be described as follows. A project is represented in activity-on-the-node representation with dummy start and end nodes. All non-dummy project activities have stochastic activity durations $\mathbf{d}_{j}$, are subject to finish start zero-lag precedence constraints and require an integer per period amount $r_{j k}$ of one or more renewable resource types $k$ $(k=1,2, \ldots K)$ during execution. All resources have a fixed per period availability $a_{k}$. Every non-dummy activity $j$ has a weight $w_{j}$ that denotes the marginal cost of deviating the realized starting time of activity $j$ during execution from its predicted activity start time in the baseline schedule. The weight of the dummy end activity $w_{n}$ denotes the cost of delaying the project completion beyond a predefined deterministic project due date $\delta_{n}$. The objective is to build a stable precedence and resource feasible baseline schedule by minimizing the stability cost function $\Sigma w_{j}\left(E\left|\mathbf{s}_{j}-s_{j}\right|\right)$, defined as the weighted sum of the expected absolute deviations between the predicted starting times $s_{j}$ of the activities in the baseline and their realized starting times $\mathbf{s}_{j}$ during actual schedule execution. Following the classification scheme of Herroelen et al. (2000) this problem can be classified as $m, 1\left|c p m, \mathbf{d}_{j}, \delta_{n}\right| \Sigma w_{j}\left(E\left|\mathbf{s}_{j}-s_{j}\right|\right)$. The first field $(m, 1)$ refers to the use of an arbitrary number of renewable resource types. The second field specifies finish-start precedence relations $(\mathrm{cpm})$, stochastic activity durations $\left(\mathbf{d}_{j}\right)$ for which the distribution function is assumed known or can be estimated, and a deterministic project due date $\left(\delta_{n}\right)$. The last field specifies the non-stability function to be minimized. The scheduling problem for stability has been shown to be ordinary NP-hard (Leus \& Herroelen 2005).

Both simple heuristics and meta-heuristics will be presented in this paper, the objective of which is to generate stable baseline schedules with acceptable makespan performance. The remainder of the paper is organized as follows. Section 2 introduces the different heuristic procedures. The set-up of the computational experiment is described in Section 3. Section 4 presents the computational results, while a last section is devoted to overall conclusions.

\section{Algorithms}

The heuristic algorithms described in this section all consider a deterministic project due date $\delta_{n}$ and start from a minimum makespan schedule in which 


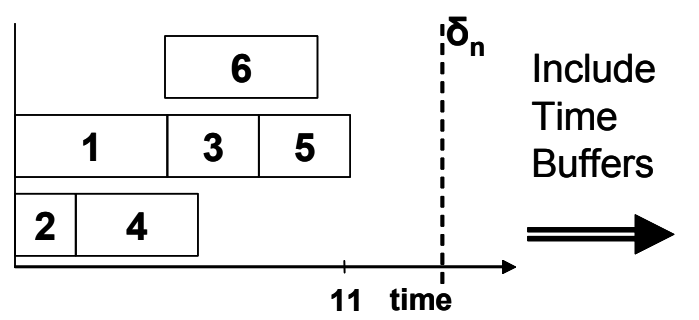

(a)

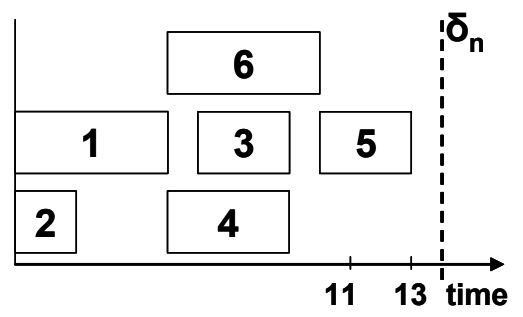

(b)

Figure 1: Inserting time buffers in a baseline schedule

time buffers are inserted in order to protect against anticipated disruptions. The minimum makespan schedule is constructed by applying the branch-andbound algorithm of Demeulemeester \& Herroelen $(1992,1997)$ for solving the deterministic resource-constrained project scheduling problem (RCPSP) using mean activity durations (problem $m, 1|c p m| C_{\max }$ (Herroelen et al. 2000)).We set $\delta_{n}=\left\lfloor 1.3 \times C_{\max }\right\rfloor$. The included time buffers are idle periods (gaps) in the schedule between the planned starting time of an activity and the latest planned finish time of its predecessors. The buffers should act as cushions to prevent propagation of a disruption throughout the schedule.

Figure 1 illustrates the insertion of time buffers in a baseline schedule. Figure 1 (a) shows a minimum makespan schedule. Figure 1(b) shows the buffered schedule with time buffers inserted in front of activities 3,4 and 5 .

Two factors are taken into consideration in determining the size of the buffer in front of an activity $i$. First, the variability of all the activities that precede activity $i$ in the schedule (measured by the standard deviation of their duration) is taken into account, because it affects the probability that activity $i$ can start at its scheduled starting time. Second, the weight of activity $i$, and both the weights of its predecessors and successors contain relevant information, because they reflect how costly it is to disrupt the starting time of activity $i$ in relation to its predecessors and successors in the schedule.

The resource flow dependent float factor (RFDFF) heuristic, proposed in Van de Vonder et al. (2004a), is used as our evaluation benchmark. This heuristic relies completely on the activity weights but does not exploit the available information offered by the activity duration distributions in making its buffering decisions. The operating principles of RFDFF will be recapitulated in Section 
2.1. An extended version RFDFF + will be presented that redefines the activity weights in terms of disruption cost and disruption probabilities. The fixed relative deviation (FRD) heuristic presented in Section 2.2, relies on the standard deviation of the duration of an activity in order to compute a modified duration to be used in constructing the baseline schedule. The starting time criticality (STC) heuristic and its variant STC+ (both are described in Section 2.3) try to combine information on activity weights and activity duration variances. Section 2.4 introduces an improvement phase that can be added to each of the just mentioned heuristics to enhance their performance. Section 2.5 describes a tabu search meta-heuristic that searches for the best buffer insertion for a given schedule by exploring the neighborhood solutions.

\section{$2.1 \quad$ RFDFF and RFDFF+}

The suboptimal resource flow dependent float factor (RFDFF) heuristic that has been developed by Van de Vonder et al. (2004a) as an extension to the adapted float factor (ADFF) heuristic proposed in Leus (2003) and Van de Vonder et al. (2004b), will serve as our benchmark. RFDFF starts from a minimum duration schedule (in this paper this is the minimum duration schedule obtained by the branch-and-bound procedure of Demeulemeester \& Herroelen $(1992,1997)$ and modifies it by adding safety buffers in front of activities. The hope is that the time buffers serve as a cushion to prohibit the propagation of the disruptions through the schedule. RFDFF+ is an extension of RFDFF.

\subsubsection{RFDFF}

The starting time of activity $j$ in the $\operatorname{RFDFF}$ schedule is calculated as $s_{j}(S):=$ $s_{j}(B \& B)+\alpha_{j}($ float $[j])$, where $s_{j}(B \& B)$ denotes the starting time of activity $j$ in the minimum duration baseline schedule and $\alpha_{j}$ denotes the activity dependent float factor. The total float, float $[j]$, is the difference between the latest allowable starting time of activity $j$ given the project due date (i.e. its starting time in the right-justified version of the minimum duration schedule) and its scheduled starting time in the minimum duration schedule.

To calculate the float factors $\alpha_{j}$, we first need to construct a resource flow network or transportation network (Artigues \& Roubellat 2000) for the minimum duration schedule. The resource flow network is a network with the same

nodes as the original project network, but with arcs connecting two nodes if there is a resource flow between the corresponding activities. It thus identifies 
how each single item of a resource is passed on between the activities in the schedule. A schedule may allow for different ways of allocating the resources so that the same schedule may be represented by different resource flow networks. We use the (random) algorithm of Artigues \& Roubellat (2000) to select a feasible resource flow network.

The float factors $\alpha_{j}$ are now calculated as $\alpha_{j}=\beta_{j} /\left(\beta_{j}+\lambda_{j}\right)$, where $\beta_{j}$ is the sum of the weight of activity $j$ and the weights of all its transitive predecessors in both the original network and the resource flow network, while $\lambda_{j}$ is the sum of the weights of all transitive successors of activity $j$ in both networks. The weights of activities that start at time 0 are not included in these summations because it is assumed that these activities can always start at their planned start time and thus do not need any buffering to cope with possible disruptions of their predecessors. The RFDFF heuristic consequently inserts longer time buffers in front of activities that would incur a high cost if started earlier or later than originally planned and resource constraints will always remain satisfied in the resulting schedule.

\subsubsection{RFDFF+}

The main disadvantage of the RFDFF heuristic is that it does not use the available information about the variability of the activity durations. Some activities are known to have a larger probability to be disrupted during project execution than others. RFDFF+ differs from RFDFF in that the activity weights are redefined to reflect the variability in activity durations. While for the basic RFDFF, $w_{i}$ equals 0 if activity $i$ can start at time 0 and otherwise equals the marginal cost $c_{i}$ of modifying the starting time of activity $i$, the calculation of the weights will be more involved for RFDFF + . For RFDFF + we set $w_{i}=c_{i} \cdot \min \left(\sum_{j:(j, i) \in\left(A \cup A^{\prime}\right)} P\left(\mathbf{d}_{j}>\left(s_{i}-s_{j}\right)\right), 1\right)$, in which $A$ denotes the arcs of the original project network and $A^{\prime}$ denotes the arcs of the resource flow network. $P\left(\mathbf{d}_{j}>\left(s_{i}-s_{j}\right)\right)$ is the probability that the disruption of predecessor $j$ will be such that the finish time of activity $j$ will delay the starting time of activity $i$, all this under the assumption that predecessor $j$ starts as planned and the current resource flow network is maintained. By summing these probabilities over all direct predecessors $\left(A \cup A^{\prime}\right)$ in the schedule and multiplying this term by the marginal cost $c_{i}$, a larger weight will be allotted to activities with a large probability to start later than planned. The RFDFF-procedure for calculating the float factors $\alpha_{i}$ is left unchanged. 


\section{$2.2 \quad$ FRD}

The fixed relative deviation (FRD) heuristic starts from a completely different point of view. Because the standard deviations $\sigma_{i}$ of the stochastic activity duration density functions are assumed known, we use this information in order to add safety time to each non-dummy activity. This is done by constructing the baseline schedule using updated activity durations $d_{i}^{\prime}=E\left(\mathbf{d}_{i}\right)+R \sigma_{i}$, where the relative deviation $R$ is activity independent and iteratively computed as follows.

At each iteration step, starting with an initial value of $0, R$ is augmented using 0.01 increments and the activity durations are set to $d_{i}^{\prime}=E\left(\mathbf{d}_{i}\right)+R \sigma_{i}$. Whenever one of the $d_{i}^{\prime}$ has changed compared to the previous step, a new baseline schedule is constructed by creating an early start schedule for the graph $G\left(N, A \cup A^{\prime}\right)$ using the updated activity durations $d_{i}^{\prime}$. By adding the original flow network precedence relations $A^{\prime}$ to the precedence network, we make sure that the resulting schedule is resource and precedence feasible. For the resulting schedule, the stability cost function $\Sigma w_{j}\left(E\left|\mathbf{s}_{j}-s_{j}\right|\right)$ is calculated and the schedule is stored if this stability cost function yields a new best solution. The algorithm stops whenever the current $R$ (and thus also all larger $R$-values) leads to a project completion time $s_{n}>\delta_{n}$.

A last remark has to be made. Often at a specific iteration step, more than one activity duration needs to be updated. Assume for example that at step $q$, $d_{1}^{\prime}=4, d_{5}^{\prime}=7$ and $d_{11}^{\prime}=4$, while at step $q+1$ these three activity durations would have to be augmented by 1 unit. In this case, iteration step $q+1$ will be split in as many substeps as there are activities for which the duration needs to be updated, and the updates are made one at a time in decreasing order of the activity weights. Thus, when for example $w_{1}=3, w_{5}=8$ and $w_{11}=2$, the first substep of step $q+1$ would construct an early start schedule by using $d_{1}^{\prime}=4$, $d_{5}^{\prime}=8$ and $d_{11}^{\prime}=4$, the second by using $d_{1}^{\prime}=5, d_{5}^{\prime}=8$ and $d_{11}^{\prime}=4$ and the last by using $d_{1}^{\prime}=5, d_{5}^{\prime}=8$ and $d_{11}^{\prime}=5$. In doing so, very valuable solutions are obtained that would have been overlooked if all activity duration updates would be made at the same time.

\section{$2.3 \quad$ STC and STC+}

The starting time criticality heuristics exploit information about both the weights of the activities and the variance structure of the activity durations. The basic idea is to start from a minimum duration schedule and iteratively create in- 
termediate schedules by adding a one-unit time buffer in front of that activity that needs it the most in the current intermediate schedule, until adding more safety would no longer improve stability. We thus need a measure to quantify for each activity how critical its current starting time is in the current intermediate baseline schedule.

The starting time criticality for activity $i$ is defined as $\operatorname{stc}[i]=P(\mathbf{s}(\mathbf{i})>$ $s(i)) \times w_{i}=\gamma_{i} \times w_{i}$, where $\gamma_{i}$ denotes the probability that activity $i$ cannot be started at its scheduled starting time.

The iterative procedure runs as follows. At each iteration step (see pseudocode below), the buffer sizes of the current intermediate schedule are updated as follows. The activities are listed in decreasing order of the $s t c[i]$. The list is scanned and the size of the buffer to be placed in front of the currently selected activity from the list is augmented by one unit and the direct and transitive successors of the activity are shifted to the right. If this new schedule has a feasible project completion $\left(<\delta_{n}\right)$ and results in a lower stability cost, the schedule will be the input schedule for the next iteration step. If not, the next activity in the list is considered. Whenever we reach to an activity $i$ for which $s t c[i]=0$ (all activities $j$ with $s(j)=0$ are by definition in this case) and no feasible improvement is found, a local optimum is obtained and the procedure terminates.

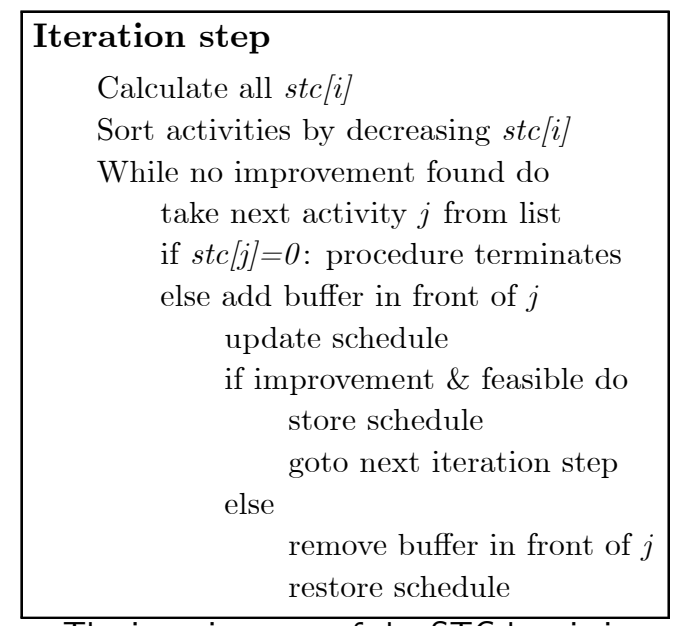

The iteration step of the STC heuristic

Regrettably, the probabilities $\gamma_{i}$ are not easy to compute. $\gamma_{i}=\bigcup_{j \in X} k(j, i)$, with $X$ being defined as the set of all direct and transitive predecessors of $i$ in both the original network and the resource flow network and where $k(j, i)=$ 
$P(\mathbf{d}(\mathbf{j})>s(i)-\mathbf{s}(\mathbf{j}))$ gives the probability that predecessor $j$ disturbs the planned starting time of activity $i$. In this expression, both $\mathbf{d}(\mathbf{j})$ and $\mathbf{s}(\mathbf{j})$ are stochastic variables. $\mathbf{s}(\mathbf{j})$ is also endogenous to the model, which makes $k(j, i)$ extremely hard to quantify. The two variants of the starting time criticality heuristic, STC and STC+, differ in their approximations for $\gamma_{i}$.

\subsubsection{STC}

STC makes two assumptions in approximating $\gamma_{i}$ : (a) only one activity at a time disturbs the starting time of activity $i$, and (b) the predecessor $j$ of activity $i$ starts at its originally planned starting time. Assumption (a) means that we estimate $\bigcup_{j \in X} k(j, i)$ by $\sum_{j \in X} k(j, i)$, i.e. we assume that $k(j 1, i) \cap k(j 2, i)=0$ for each $j 1, j 2 \in X$. Assumption (b) boils down to setting $\mathbf{s}(\mathbf{j})=s(j)$. Combining both assumptions yields $\gamma_{i}^{\prime}=\sum_{j \in X} P(\mathbf{d}(\mathbf{j})>s(i)-s(j))$ such that $s t c[i]=\gamma_{i}^{\prime} \times w_{i}$.

\subsubsection{STC +}

STC was based on the assumption (b) that each of the predecessors of activity $i$ starts as planned, i.e. $P(\mathbf{s}(\mathbf{j})>s(j))=0$. Because we assume in this paper that during execution activities are not allowed to start before their planned starting time $(P(\mathbf{s}(\mathbf{j})<s(j))=0)$, we know by definition of $\gamma_{j}$ that $P(\mathbf{s}(\mathbf{j})=$ $s(j))=1-\gamma_{j}$. Given that all starting times are considered discrete, an exact calculation of $k(j, i)$ would be: $k(j, i)=P(\mathbf{d}(\mathbf{j})>s(i)-\mathbf{s}(\mathbf{j}))$

$$
\begin{aligned}
= & \sum_{q=0}^{q \rightarrow \infty} P(\mathbf{d}(\mathbf{j})>s(i)-(s(j)+q)) \times P(\mathbf{s}(\mathbf{j})=s(j)+q) \\
= & P(\mathbf{d}(\mathbf{j})>s(i)-s(j)) \times P(\mathbf{s}(\mathbf{j})=s(j)) \\
& +\sum_{q \rightarrow \infty} P(\mathbf{d}(\mathbf{j})>s(i)-(s(j)+q)) \times P(\mathbf{s}(\mathbf{j})=s(j)+q) \\
\approx & P(\mathbf{d}(\mathbf{j})>s(i)-s(j))\left(1-\gamma_{j}\right)+P(\mathbf{d}(\mathbf{j})>s(i)-(s(j)+1)) \gamma_{j},
\end{aligned}
$$

making the assumption that $P(\mathbf{s}(\mathbf{j})>s(j)+1)=0$, which is obviously less strict than the original second assumption that $P(\mathbf{s}(\mathbf{j})>s(j))=0$.

We know that $\gamma_{i}=\sum_{j \in X} k(j, i) \cdot \gamma_{i}$ is approximated as

$$
\gamma^{\prime \prime}{ }_{i}=\sum_{j \in X} P(\mathbf{d}(\mathbf{j})>s(i)-s(j))\left(1-\gamma^{\prime \prime}{ }_{j}\right)+P(\mathbf{d}(\mathbf{j})>s(i)-(s(j)+1)) \gamma_{j}{ }^{\prime} .
$$

If we make sure that the starting time criticalities $s t c[i]$ are calculated in increasing activity starting time order, the values of $\gamma_{j}^{\prime \prime}$ have already been calculated for each predecessor $j$ of $i$ when we calculate $\gamma_{i}^{\prime \prime}$. 


\subsection{Improvement heuristic}

The improvement heuristic starts from an initial solution. This can be the schedule found by any of the heuristics discussed above. The activities are entered in a list in decreasing order of their starting time in the input schedule. The activities are considered in the order dictated by the list. For the currently selected activity from the list, it is determined how many periods the activity can be moved backward and forward in time without affecting the starting time of any other activity in the schedule. From all discrete time instants in this displacement interval, the instant that yields the lowest stability cost during simulation, is chosen as the new starting time of the current activity in the updated schedule. With this updated schedule, we proceed to the next activity in the list. If the next activity in the list is the dummy start activity, we restart the list. If the list is scanned entirely without any improvement, a local optimum has been found and the procedure terminates.

Basically the algorithm is a combination of steepest and fastest descent. For an activity selected from the list, we examine all possible starting times and select the best one (steepest descent). However, we do not examine the entire range of starting times of all the activities and select the best, but instead we already update the schedule if a better solution is found for the current activity before proceeding to the next activity in the list. This fastest descent part of the algorithm is included to speed up computations.

\subsection{Tabu search}

Descent approaches may terminate at a local optimum after some iterations when no further improvement can be found in the direct neighborhood of the current solution. Glover $(1989,1990)$ developed the principle of tabu search algorithms, which allow to select the mildest ascent solution to continue the search whenever no improvement can be found. A tabu list keeps track of recent solutions that will be forbidden moves in order to avoid cycling.

The tabu search procedure departs from the FRD-schedule described in Section 2.2. At each iteration step, the neighborhood of the current solution contains at most $2 \times(n-2)$ solutions. For each non-dummy activity of the project, we have two possible neighborhood solutions. One is obtained by increasing the buffer in front of the activity in the schedule by one time period, if possible (plus-move). The other is obtained by decreasing the buffer size of this activity by one unit, if possible (minus-move). The buffers in front of all other activities 
are left unchanged. Two tabu lists are kept, both of length $n / 3$. The first list stores all recent plus-moves, while the second one stores all recent minus-moves. Before allowing a new plus-move, we have to check whether this activity is not in the second list. If a buffer size decrease (minus-move) delivers the best solution in the neighborhood, the first tabu list has to be checked. By doing so, we avoid cycling, but we do allow an activity to be consecutively selected more than once if the considered moves have the same direction. The aspiration criterion defines that a move that would yield a new best solution will be accepted even if it would normally be prohibited by the tabu list. Because the tabu search described here only adds or subtracts one unit of time buffer at a time, large shifts of the starting time of an activity compared to its initial starting time will only occur if all intermediate positions yield acceptable solutions. This might obstruct the procedure to move an activity into its actual best positioning for all other activity starting times considered fixed. To remove this inconvenience, one iteration step of the improvement heuristic of Section 2.4 will be allowed after a fixed number of iterations (100). The overall best found solution is stored throughout the whole procedure. The tabu search stops after a fixed number of iterations.

\section{Experimental set-up}

All proposed algorithms have been coded in Microsoft Visual $\mathrm{C}++$ 6.0. The procedures were tested on two data sets. A first set consist of the 480 networks of the J30 instance set of PSPLIB (Kolisch \& Sprecher 1997). A second set consists of the eighty 30-activity networks constructed in Van de Vonder et al. (2005) by using the RanGen project scheduling network instances generator developed by Demeulemeester et al. (2003) using two settings (0.5 and 0.75) for the order strength, resource factor and resource constrainedness. For an extensive study of the impact of the parameter settings on schedule stability we refer to Van de Vonder et al. (2004a).

In order to investigate the impact of activity duration variability, we distinguish between low, high and random duration variability. High duration variability means that the real activity durations are all discretized values drawn from

a right-skewed beta-distribution with parameters 2 and 5, that is transformed in such a way that the minimum duration equals 0.25 times the expected duration, the mean duration equals the expected duration and the maximum duration 


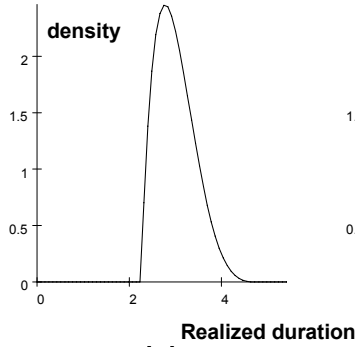

(a)

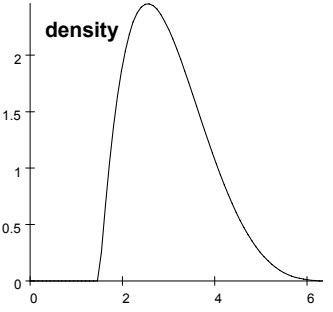

(b)

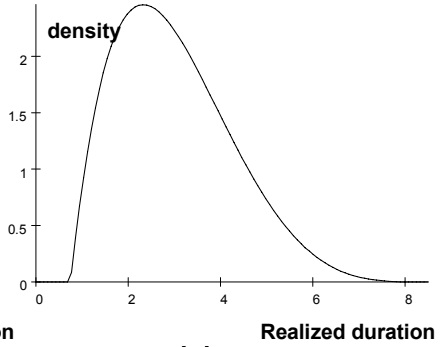

(c)

Figure 2: Distribution functions for low (a), medium (b) and high (c) duration variability if $E\left(d_{i}\right)=3$

equals 2.875 times the expected duration. Low duration variability means that the realized activity durations are also discretizations of values drawn from a beta-distribution with parameters 2 and 5, but with the mean equal to the expected activity duration and with minimum and maximum values equal to 0.75 times and 1.625 times the expected activity duration, respectively. Last, random duration variability stands for the case where no overall uncertainty level exists for the project and the variabilities are activity dependent. We randomly select for every activity whether the activity has high, low or medium duration variability. The distribution functions for high and low variability are as explained above, while medium duration variability is an intermediate case where the realized activity durations are drawn from a beta-distribution with parameters 2 and 5, but with minimum and maximum values equal to 0.5 times and 2.25 times the expected activity duration, respectively. Figure 2 shows the distribution functions from which the realized durations are drawn for an activity with an expected 3-period duration.

The stability cost $\Sigma w_{j}\left(E\left|\mathbf{s}_{j}-s_{j}\right|\right.$ is evaluated by drawing the $w_{j}$ for each non-dummy activity $j \in\{1,2 \ldots . n-1\}$ from a discrete triangular distribution with $P\left(w_{j}=q\right)=(21-2 q) \%$ for $q \in\{1,2 \ldots .10\}$. This distribution results in a higher probability for low weights and in an average weight $w_{\text {avg }}=3.85$. The weight $w_{n}$ of the dummy end activity denotes the marginal cost of not making the projected project completion and will be fixed at $\left\lfloor 10 \times w_{\text {avg }}\right\rfloor=38$. For an extensive evaluation of the impact of the activity weight of the dummy end activity, we refer to Van de Vonder et al (2004a, 2005)).

Extensive simulation will be used to evaluate all procedures on stability and 
computational efficiency. For every network instance, two sets of 100 executions (referred to further on as the training set and the test set) are simulated by drawing different actual activity durations from the described distribution functions. The test set of executions is run to avoid overfitting as will be explained below.

Using the simulated activity durations, the realized schedule is constructed by applying the following reactive procedure. First, an activity list is deduced from the baseline schedule by ordering the activities in increasing order of their starting time. Ties are broken by decreasing order of activity weight, then by increasing activity number. Afterwards, the realized schedule is constructed by applying a parallel schedule generation scheme (SGS) on this activity list using the actual activity durations. To maintain stability, an activity is never allowed to start earlier than its scheduled starting time. Thus, at each decision point we scan the ordered activity list to select the set of eligible unscheduled activities that have a baseline starting time that is not larger than the current decision time. It should be observed that the resource allocation (i.e. the flow network) of the resulting realized schedule and the baseline schedule may differ. For a comparison of different reactive procedures we refer the reader to Van de Vonder et al. (2005).

The algorithms proposed in Sections 2.4 and 2.5 select the best neighborhood solution drawing disruptions from the stochastic activity duration density functions. Including information about these simulated disruptions might make the buffer allocation decision process subject to overfitting. The best schedule for the simulated disruptions will not necessarily be the best schedule for the actual disruptions during project execution, even if we assume that they are drawn from the same density functions. As mentioned above, we try to avoid overfitting by examining the results on both the training and test set of execution scenarios.

\section{Computational results}

All computational results have been obtained on a Pentium IV $2.4 \mathrm{GHz}$ personal computer. A total of fourteen scheduling procedures are evaluated. The minimum duration schedule obtained by the branch-and-bound procedure of Demeulemeester \& Herroelen $(1992,1997)$ serves as the benchmark. Algorithms 2-6 are the RFDFF, RFDFF +, FRD, STC and STC+ heuristics introduced in 
Sections 2.1-2.3. Algorithms 7-12 are all instances of the improvement heuristic of Section 2.4 with the solutions of algorithms 1-6 as the initial solution. The tabu search procedure of Section 2.5 is executed with 100 (Algorithm 13) and 300 (Algorithm 14) iteration steps, respectively. Algorithms 1-6 will be referred to as simple heuristics, while algorithms 7-14 will be called the improvement heuristics.

For every algorithm we calculate the average stability cost (Stab) over all networks and executions on both a training set and a test set of simulated disruptions. \%Best denotes the percentage of network instances for which a certain algorithm yields the minimum stability cost among the algorithms within its class. Again, this measure will be calculated for training and test set disruptions to examine the degree of overfitting. We feel that comparing simple heuristics with improvement algorithms would give few additional insights. Finally, also the average computational times (in seconds) are given for each algorithm. Note that the computational time of algorithms 2-14 include the computational time of algorithm 1, because the RCPSP always needs to be solved. Also, the reported computational times of the improvement heuristics 8-14 will comprise the time to calculate their initial solution.

In the remainder of this section, results will be discussed for the three activity duration variability cases introduced in the previous section.

\subsection{High duration variability}

Tables 1 and 2 show the average results on the PSPLIB and RanGen data set when all activities are subject to high activity duration variability.

\subsubsection{Simple heuristics}

Tables 1 and 2 reveal that STC+ gives the best results among the simple heuristics, closely followed by STC. Although FRD, RFDFF and RFDFF+ have a much smaller stability cost than the minimum duration schedule, they only outperform STC+ and STC on a few network instances.

Remarkably, the newly proposed RFDFF+ heuristic does not yield a better average stability cost than RFDFF. We explained in Section 2.1.2 that the procedure used to calculate the weights $w_{i}$ in RFDFF+ uses additional information on the variance structure compared to RFDFF. Better weights are expected due to this more complete information, but RFDFF+ shows major difficulties in calculating an appropriate weight $w_{n}$ for the dummy end activity. This will 


\begin{tabular}{lccccc}
\hline & Training Set & Test Set & \\
\hline Algorithm & Stab & \%Best & Stab & \%Best & Time \\
\hline 1. RCPSP & 400.68 & 0 & 400.97 & 0 & 0.12 \\
2. RFDFF & 137.14 & 7 & 137.70 & 7 & 0.13 \\
3. RFDFF+ & 142.31 & 3 & 142.93 & 4 & 0.13 \\
4. FRD & 145.53 & 6 & 147.66 & 5 & 0.32 \\
5. STC & 124.18 & 40 & 124.86 & 42 & 0.16 \\
6. STC + & 122.68 & 44 & 123.70 & 43 & 0.17 \\
\hline 7. RCPSP D & 107.22 & 2 & 115.66 & 8 & 3.95 \\
8. RFDFF D & 99.90 & 11 & 107.86 & 16 & 2.52 \\
9. RFDFF+ D & 100.37 & 12 & 108.54 & 13 & 2.61 \\
10. FRD D & 99.66 & 9 & 107.82 & 13 & 2.86 \\
11. STC D & 100.73 & 9 & 108.59 & 15 & 2.54 \\
12. STC+ D & 100.28 & 12 & 107.98 & 19 & 2.49 \\
13. Tabu 100 & 98.60 & 27 & 107.70 & 17 & 17.33 \\
14. Tabu 300 & 98.02 & 64 & 106.80 & 25 & 50.57 \\
\hline 15. Best & 97.56 & 100 & 104.48 & 100 & \\
\hline
\end{tabular}

Table 1: Results on PSPLIB data set with high duration variability

\begin{tabular}{llllll}
\hline & Training Set & \multicolumn{2}{l}{ Test Set } & \\
\hline Algorithm & Stab & \%Best & Stab & \%Best & Time \\
\hline 1. RCPSP & 558.62 & 0 & 555.81 & 0 & 0.02 \\
2. RFDFF & 155.94 & 1 & 154.70 & 0 & 0.03 \\
3. RFDFF+ & 157.15 & 1 & 155.84 & 5 & 0.03 \\
4. FRD & 143.33 & 19 & 144.95 & 20 & 2.23 \\
5. STC & 136.10 & 28 & 136.68 & 25 & 0.07 \\
6. STC + & 134.28 & 51 & 134.29 & 51 & 0.07 \\
\hline 7. RCPSP D & 127.19 & 1 & 134.13 & 6 & 5.84 \\
8. RFDFF D & 114.13 & 4 & 121.5 & 11 & 2.73 \\
9. RFDFF+ D & 113.99 & 6 & 120.85 & 15 & 2.75 \\
10. FRD D & 111.76 & 9 & 119.02 & 19 & 2.46 \\
11. STC D & 113.00 & 3 & 120.62 & 13 & 2.70 \\
12. STC+ D & 112.94 & 5 & 120.97 & 16 & 2.68 \\
13. Tabu 100 & 110.46 & 47 & 118.76 & 22 & 17.26 \\
14. Tabu 300 & 109.83 & 78 & 118.34 & 23 & 51.13 \\
\hline 15. Best & 109.50 & 100 & 116.07 & 100 & \\
\hline
\end{tabular}

Table 2: Results on RanGen data set with high duration variability 
result in a project buffer size being either too small or too big, both cases leading to an increase in stability cost.

On average, FRD shows worse results than both RFDFF variants on the PSPLIB set, but surprisingly generates better results on the RanGen problem set. This is due to the differences in the experimental designs used to construct both data sets. For all the networks in the RanGen data set a rather high resource factor and resource constrainedness (0.5 or 0.75$)$ are assumed, resulting in resource intensive networks with many forbidden sets. On the other hand, the factorial design for PSPLIB also includes networks that are less resource constrained.

When we only select the 60 resource intensive networks with resource factor exceeding 0.5 and resource strength equal to 0.2 from the PSPLIB data set, we observe that the average stability cost of the FRD heuristic equals 173.91, which is significantly better than the stability costs of RFDFF (189.96) and RFDFF+ (190.00). We might thus conclude that RFDFF and RFDFF+ encounter problems when dealing with resource intensive networks. In these networks many resource conflicts need to be resolved, which will lead to more extra precedence relations in the resource flow network and eventually to a larger $C_{\max }$ for the RCPSP and thus a larger $\delta_{n}$. RFDFF and RFDFF+ typically allocate a larger portion of the total safety to the dummy end activity than the other heuristics. For resource intensive networks, the total safety included (recall that $\left.\delta_{n}=\left\lfloor 1.3 \times C_{\text {max }}\right\rfloor\right)$ might be too high such that RFDFF and RFDFF + overprotect the project completion and have to pay a high extra stability cost for this unnecessary extra protection due to poorly buffered intermediate activities. This explains the comparative stability advantage of FRD on resource intensive data sets, such as the 80 networks of the RanGen data set and the 60 selected networks of PSPLIB.

The required computational effort is highly correlated with the number of time-consuming ${ }^{1}$ evaluations needed in the procedure, which is low for all simple heuristics. Because FRD selects the best among a range of solutions, its computational time is the most demanding among the simple heuristics. For the same reason, FRD might be slightly affected by overfitting. Indeed, if there is an increase in average stability cost in the test set over the training set results, it is more pronounced for FRD than for the other simple heuristics. However, this overfitting is clearly not critical.

\footnotetext{
${ }^{1}$ Remember that each evaluation consists of 100 simulated executions of the project that have to be scheduled by using the parallel SGS.
} 


\subsubsection{Improvement heuristics}

Comparing algorithms 7-12 on the training set reveals that all stable project schedules (2-5) provide good starting solutions for the improvement heuristic of Section 2.4. Only the minimum duration schedule leads to a substantial stability cost and computational effort. Surprisingly, FRD (and not STC) provides the overall best initial solution. Contrary to all other heuristics in this paper, FRD does not include any information about activity weights in the buffer allocation process. As a result, buffers are typically spread more equally over the activities leading to time buffers being inserted in front and behind most of the activities and resulting in more possible search directions for descent approaches. This directly explains why the FRD schedule has been chosen as initial solution for the tabu search. As somewhat expected, tabu search obtains the best results of all heuristics.

Row 15 represents the average over all networks of the best solutions found by any of the 14 algorithms. In $64 \%$ of the PSPLIB networks and $78 \%$ of the RanGen networks, this best solution has been obtained by Tabu 300. The deviation between the average stability cost of Tabu 300 and the stability cost of row 15 is lower than $0.5 \%$ for both data sets. The $2 \times(n-2)$ evaluations required at each of the 300 (100) iteration steps of the tabu search procedure account for the increase in computation time.

When comparing the performance of the improvement algorithms on the test set, we observe an increase in the average stability costs, while the mutual differences in stability cost between the algorithms are largely reduced. Although Tabu 300 remains slightly better than FRD D on as well Stab as \%Best, the small differences make it harder to justify the high computational burden of Tabu 300. We also note that the performance deviation of Tabu 300 from the best result found over all 14 algorithms has increased from less than $0.5 \%$ to approximately $2 \%$. We may conclude that the tabu search is subject to overfitting.

\subsection{Low duration variability}

Recent research (Van de Vonder et al. (2005)) showed that when activity duration variability is rather low, proactive scheduling becomes increasingly attractive because the disadvantage in makespan performance compared to a minimum duration schedule becomes very small, while the improvement on stability remains large. 


\begin{tabular}{lccccc}
\hline & \multicolumn{2}{l}{ Training Set } & Test Set & \\
\hline Algorithm & Stab & \%Best & Stab & \%Best & Time \\
\hline 1. RCPSP & 123.17 & 0 & 122.64 & 0 & 0.12 \\
2. RFDFF & 6.11 & 0 & 6.01 & 0 & 0.12 \\
3. RFDFF+ & 7.37 & 0 & 7.25 & 0 & 0.13 \\
4. FRD & 1.84 & 9 & 1.97 & 7 & 0.28 \\
5. STC & 1.32 & 59 & 1.28 & 60 & 0.16 \\
6. STC + & 1.06 & 65 & 1.06 & 66 & 0.17 \\
\hline 7. RCPSP D & 6.15 & 0 & 7.03 & 0 & 3.38 \\
8. RFDFF D & 1.25 & 5 & 1.72 & 4 & 1.71 \\
9. RFDFF+ D & 1.57 & 1 & 2.04 & 5 & 1.82 \\
10. FRD D & 0.78 & 5 & 1.29 & 5 & 1.39 \\
11. STC D & 0.75 & 31 & 1.14 & 23 & 1.13 \\
12. STC+ D & 0.68 & 29 & 1.05 & 26 & 1.13 \\
13. Tabu 100 & 0.60 & 48 & 1.17 & 13 & 12.10 \\
14. Tabu 300 & 0.56 & 77 & 1.19 & 17 & 35.58 \\
\hline 15. Best & 0.52 & 100 & 0.78 & 100 & \\
\hline
\end{tabular}

Table 3: Results on PSPLIB data set with low duration variability

\begin{tabular}{lccccc}
\hline & \multicolumn{2}{l}{ Training Set } & Test Set & \\
\hline Algorithm & Stab & \%Best & Stab & \%Best & Time \\
\hline 1. RCPSP & 172.92 & 0 & 171.46 & 0 & 0.02 \\
2. RFDFF & 7.09 & 0 & 6.89 & 0 & 0.02 \\
3. RFDFF+ & 7.36 & 0 & 7.35 & 0 & 0.03 \\
4. FRD & 1.88 & 11 & 1.88 & 10 & 0.18 \\
5. STC & 1.19 & 58 & 1.27 & 62 & 0.07 \\
6. STC + & 1.03 & 72 & 1.05 & 67 & 0.08 \\
\hline 7. RCPSP D & 22.00 & 0 & 22.74 & 0 & 3.89 \\
8. RFDFF D & 2.34 & 0 & 2.69 & 0 & 1.48 \\
9. RFDFF+ D & 2.55 & 3 & 3.10 & 0 & 1.49 \\
10. FRD D & 0.85 & 9 & 1.27 & 10 & 1.19 \\
11. STC D & 0.78 & 28 & 1.11 & 27 & 0.87 \\
12. STC+ D & 0.75 & 25 & 1.06 & 24 & 0.86 \\
13. Tabu 100 & 0.69 & 49 & 1.22 & 13 & 12.11 \\
14. Tabu 300 & 0.66 & 73 & 1.24 & 11 & 36.58 \\
\hline 15. Best & 0.64 & 100 & 0.85 & 100 & \\
\hline
\end{tabular}

Table 4: Results on RanGen data set with low duration variability 


\subsubsection{Simple heuristics}

Tables 3 and 4 give an overview of the obtained results. Compared to the results of Section 4.1 we observe that RFDFF+ performs relatively worse on the training set and is even dominated $(\%$ Best $=0)$ on all networks by the other simple heuristics. RFDFF does not use any information about activity duration variability and will thus construct exactly the same schedule whatever the amount of uncertainty in the environment. While this procedure provides reasonable results in the case of high variability, its stability cost in the case of low variability is not competitive with the stability cost values obtained by other simple heuristics. As already described above, RFDFF + overprotects the project completion, causing poorly buffered intermediate activities and unnecessary stability losses during execution.

STC + ranks once again the best among the simple heuristics, closely followed by STC and FRD. Taking into account that $w_{\text {avg }}=3.85$, their low average stability costs $(<2)$ indicate that almost every activity of the project will be executed as planned by applying these buffer allocation procedures without any loss in makespan performance compared to the RCPSP solution.

The results obtained on the test set are very similar. We only note a substantial smaller \%Best performance for FRD. Because FRD evaluates multiple solutions and selects the best one, it does not come as a surprise that this heuristic is somewhat subject to overfitting, while all other simple heuristics do not use any evaluation for buffer allocation and are by definition unaffected by overfitting.

\subsubsection{Improvement algorithms}

Improvement algorithms 10-14 all generate extremely satisfying results. Tabu 300 again ranks best among all heuristics on the training set. Contrary to the high variability case, STC + D obtains a slightly smaller average stability cost than FRD D on both the training and test set. On the test set, we remark that STC+ D outperforms Tabu 300 on as well Stab as \%Best. Even the simple heuristic STC+ without improvement phase generates a lower average stability cost than Tabu 300 and for the RanGen data set even the most stable schedule. The improvement phase in STC+ D has almost no impact. The more intensive local search done by the tabu search algorithm only overfits the baseline schedule on the simulated disruptions in the training set. Tabu search is certainly not recommended in the low duration variability case. 


\begin{tabular}{lccccc}
\hline & \multicolumn{2}{l}{ Training Set } & \multicolumn{2}{l}{ Test Set } & \\
\hline Algorithm & Stab & \%Best & Stab & \%Best & Time \\
\hline 1. RCPSP & 267.14 & 0 & 267.81 & 0 & 0.12 \\
2. RFDFF & 58.00 & 2 & 57.76 & 1 & 0.12 \\
3. RFDFF+ & 60.14 & 2 & 59.97 & 2 & 0.13 \\
4. FRD & 57.83 & 3 & 58.13 & 4 & 0.29 \\
5. STC & 43.37 & 44 & 43.27 & 41 & 0.16 \\
6. STC + & 42.53 & 49 & 42.33 & 53 & 0.17 \\
\hline 7. RCPSP D & 36.08 & 3 & 39.63 & 6 & 3.65 \\
8. RFDFF D & 32.12 & 9 & 35.26 & 11 & 2.27 \\
9. RFDFF+ D & 32.29 & 10 & 35.56 & 13 & 2.30 \\
10. FRD D & 31.70 & 6 & 34.97 & 9 & 2.46 \\
11. STC D & 31.98 & 15 & 35.05 & 18 & 2.05 \\
12. STC+ D & 31.81 & 14 & 34.74 & 20 & 2.04 \\
13. Tabu 100 & 30.93 & 31 & 34.70 & 18 & 15.53 \\
14. Tabu 300 & 30.55 & 67 & 34.21 & 28 & 45.21 \\
\hline 15. Best & 30.21 & 100 & 32.78 & 100 & \\
\hline
\end{tabular}

Table 5: Results on PSPLIB data set with random duration variability

\begin{tabular}{lccccc}
\hline & \multicolumn{2}{l}{ Training Set } & Test Set & \\
\hline Algorithm & Stab & \%Best & Stab & \%Best & Time \\
\hline 1. RCPSP & 397.57 & 0 & 374.91 & 0 & 0.02 \\
2. RFDFF & 65.46 & 0 & 64.75 & 0 & 0.03 \\
3. RFDFF+ & 62.27 & 1 & 60.97 & 3 & 0.03 \\
4. FRD & 55.41 & 8 & 54.90 & 6 & 0.20 \\
5. STC & 43.16 & 36 & 42.29 & 41 & 0.07 \\
6. STC + & 42.62 & 56 & 41.63 & 53 & 0.08 \\
\hline 7. RCPSP D & 48.31 & 0 & 50.58 & 3 & 6.07 \\
8. RFDFF D & 36.79 & 6 & 39.08 & 4 & 2.40 \\
9. RFDFF+ D & 35.82 & 10 & 37.92 & 13 & 2.32 \\
10. FRD D & 34.36 & 6 & 37.00 & 15 & 2.11 \\
11. STC D & 34.43 & 11 & 36.75 & 19 & 1.86 \\
12. STC+ D & 34.26 & 14 & 36.42 & 20 & 1.75 \\
13. Tabu 100 & 32.93 & 30 & 35.77 & 21 & 15.45 \\
14. Tabu 300 & 32.64 & 63 & 35.50 & 24 & 45.79 \\
\hline 15. Best & 32.29 & 100 & 34.23 & 100 & \\
\hline
\end{tabular}

Table 6: Results on RanGen data set with random duration variability 


\subsection{Random duration variability}

In this case, we assume that the project manager is able to estimate the amount of uncertainty present in each individual activity. By taking this information into account when allocating buffers, RFDFF+, FRD, STC and STC+ construct baseline schedules that should be better adapted to the project under consideration.

Overall, the obtained results (Tables 5 and 6 ) are very similar to the ones obtained for the high duration variability case of Section 4.1. First, STC+ is again the best performing simple heuristic on the PSPLIB and RanGen data sets. Second, FRD offers again a satisfying initial solution for the improvement heuristic. However, the results of Tables 5 and 6 show that STC D and STC+ D obtain comparable results on both the training and the test set. Similar to the high duration variability case, the tabu search procedure obtains the best overall results, although the differences between training set and test set indicate that they are partially affected by overfitting.

The RFDFF schedule that does not use the available extra information, performs on average slightly worse than FRD on PSPLIB, while its disadvantage becomes even more explicit on the resource intensive RanGen networks. In Table 6 we see that on these networks, the weight adaptation of RFDFF + turns out to be an improvement. However, RFDFF + is outperformed by the other simple heuristics.

We might conclude that $\mathrm{STC}(+)(\mathrm{D})$ manages best to deal with random duration variability. Apart from the tabu search procedure, we see few reasons to use other heuristics than $\mathrm{STC}(+)(\mathrm{D})$ in the random variability case. However, extra examination of the results revealed that FRD still provides a (negligible) better starting solution for tabu search than STC+.

\section{Conclusions and further research}

Proactive project scheduling is concerned with building stable baseline schedules that are able to absorb most of the anticipated disruptions during project execution. In this paper, we presented various heuristic algorithms for inserting time buffers in a project schedule.

An extensive simulation-based experiment on PSPLIB and RanGen instances revealed that the $\mathrm{STC}+$ heuristic in general ranks best among the simple heuristics that do not rely on an improvement phase. STC+ uses information on ac- 
tivity weights and activity duration variability for the buffer allocation process.

Improvement heuristics will typically yield better solutions, but may be subject to overfitting. To avoid this, results were examined on both a training and a test set, where the test set was constructed by drawing different actual activity durations from the distribution function. When the project environment comprises low activity duration uncertainty, any improvement on the STC+ baseline schedule will very likely turn out to be overfitting during project execution. When some or all project activities are subject to considerable uncertainty, local search will improve the stability of the baseline schedule even after the impact of overfitting is removed. The proposed tabu search procedure results in the minimum expected cost of the project, but is rather time consuming. A descent approach with STC+ or FRD as initial scheduling procedure results in an almost equally small expected stability cost and requires much less computational effort to calculate the baseline schedule.

In this paper buffers were heuristically allocated to a given minimum duration schedule while the resource allocation was kept fixed. The development of efficient exact buffer allocation procedures is a topic for further research. The study of the impact of different initial schedules and different resource allocations on the efficiency and effectiveness of the buffer allocation process is also an interesting open research issue.

\section{Acknowledgements}

This research has been supported by Project OT/03/14 of the Research Fund K.U.Leuven.

\section{References}

Artigues, C. \& Roubellat, F. (2000). A polynomial activity insertion algorithm in a multi-resource schedule with cumulative constraints and multiple modes. European Journal of Operational Research, 127, pp 294-316.

Demeulemeester, E. \& Herroelen, W. (1992). A branch-and-bound procedure for the multiple resource-constrained project scheduling problem. Management Science, 38, pp 1803-1818. 
Demeulemeester, E. \& Herroelen, W. (1997). New benchmark results for the resource-constrained project scheduling problem. Management Science, 43, pp 1485-1492.

Demeulemeester, E., Vanhoucke, M. \& Herroelen, W. (2003). RanGen: A random network generator for activity-on-the-node networks. Journal of Scheduling, 6, pp 17-38.

Glover, F. (1989). Tabu search, Part I. INFORMS, Journal of Computing, 1, pp 190-206.

Glover, F. (1990). Tabu search, Part II. INFORMS, Journal of Computing, 2 , pp 4-32.

Herroelen, W., De Reyck, B. \& Demeulemeester, E. (2000). On the paper "Resource-constrained project scheduling: notation, classification, models and methods" by Brucker et al.. European Journal of Operational Research, 128(3), pp 221-230.

Kolisch, R. \& Sprecher, A. (1997). PSPLIB - a project scheduling library. European Journal of Operational Research, 96, pp 205-216.

Leus, R. (2003). The generation of stable project plans. PhD thesis. Department of applied economics, Katholieke Universiteit Leuven, Belgium.

Leus, R. \& Herroelen, W. (2005). The complexity of machine scheduling for stability with a single disrupted job. Operations Research Letters, 33, pp 151156.

Van de Vonder, S., Demeulemeester, E. \& Herroelen, W. (2005). An investigation of efficient and effective predictive-reactive project scheduling procedures. Research Report 0466. Department of applied economics, Katholieke Universiteit Leuven, Belgium.

Van de Vonder, S., Demeulemeester, E., Herroelen, W. \& Leus, R. (2004a). The trade-off between stability and makespan in resource-constrained project scheduling. Research Report 0423. Department of applied economics, Katholieke Universiteit Leuven, Belgium.

Van de Vonder, S., Demeulemeester, E., Herroelen, W. \& Leus, R. (2004b). The use of buffers in project management: the trade-off between stability and makespan. International Journal of Production Economics, to appear. 